\title{
Demand dispatch in cyber-physical load aggregation system with multilevel incentives
}

\author{
Lukun ZENG ${ }^{1}$, Yi SUN ${ }^{1}$, Xingjie ZHOU ${ }^{1}$, Bin $\mathrm{LI}^{\mathbf{1}}, \operatorname{Bing}_{\mathrm{QI}}{ }^{\mathbf{1}}$
}

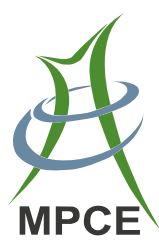

\begin{abstract}
This paper presents a demand dispatch strategy of aggregated electric water heaters (EWHs) for a load aggregation system at demand side, based on the theory of cyber-physical system. The objective is to solve the problem of water heater load control when the cyber-physical load aggregation system participates in demand dispatch of the power grid. First, an implementation framework of the demand dispatch strategy is designed between the cyber space and the physical space, including state awareness, real-time analysis, scientific decision-making and precise execution. Second, a multilevel incentive model, an EWH appliance model and a thermostat setpoint control rule are introduced. Next, based on the models and the rule, the state awareness logic, real-time analysis logic, scientific decision-making logic and precise execution logic of the strategy are designed to implement demand dispatch of aggregated EWHs. Finally, simulation results confirm the
\end{abstract}

CrossCheck date: 27 July 2018

Received: 22 November 2017/Accepted: 27 July 2018/Published online: 8 September 2018

(c) The Author(s) 2018

$\triangle$ Lukun ZENG

13811528862@163.com

Yi SUN

sy@ncepu.edu.cn

Xingjie ZHOU

1458197578@qq.com

Bin LI

direfish@163.com

Bing QI

bellqi@sina.com

1 School of Electrical and Electronic Engineering, North China Electric Power University, Beijing 102206, China effectiveness, the advantage and excellent scalability of the proposed strategy.

Keywords Electric water heater, Cyber-physical system, Load aggregation system, Demand dispatch

\section{Introduction}

Demand response (DR) traditionally refers to inducing customers to curtail electric demand by dynamic pricing or incentives at peak times to alleviate the conflict between the power supply and demand [1-3]. As more and more intermittent and fluctuating renewable generation resources have been integrated into the power grid, the situation of supply exceeding demand may occur at any time, which requires the increasement of electric demand or the curtailment of generation energy to balance demand and supply $[4,5]$. It has given rise to a new way of thinking about DR, which is called demand dispatch.

Demand dispatch is the capability to aggregate and precisely control (or dispatch) individual loads on command. Compared with traditional DR, it can not only turn off loads to reduce demand, but also turn on loads to increase demand. Moreover, it can be deployed anytime, not just at peak times. Therefore, it is deemed to be a generalization and extension of DR [6].

The residential loads represent a significant part in electric energy demand $[7,8]$. The single load is unable to participate in the supply-demand regulation of power grid for limited regulation capability. Therefore, demand dispatch is one of the effective approaches for residential loads to participate in the supply-demand regulation. Among all residential loads, electric water heaters (EWHs) are good candidates for demand dispatch. The reasons are 
mainly based on the following three aspects. First, EWHs can store electric energy by heating the hot water in their water tank [9]. Second, aggregated EWHs have a large adjustable capacity for their high popularity rate and enormous number at users' side [10]. Third, the new-generation EWH equipment has realized the integration of perception, calculation, control and communication function, which is advantageous to realize automatic control.

In the literature, there have been many relevant studies on demand dispatch for aggregated EWHs. Ernest Orlando Lawrence Berkeley National Laboratory has developed the open automated demand response (OpenADR) communications specification [11-13], which provides an open standards-based communications data model and enables demand dispatch to be fully automated. In [14], the authors propose a multilevel incentive model for demand aggregation systems of residential loads. In $[15,16]$, a timediscrete EWH load model is presented, which provides a digital mapping model of the real EWH appliance in the cyber space. In [17], a state-queueing model is developed to analyze the price response of aggregated thermostatically controlled loads (TCLs). Based on the state-queueing model, the authors put forward model-driven control strategies of aggregated TCLs for demand dispatch $[18,19]$.

With the deep and pervasive application of information and communication technologies, load aggregation systems which are involved in power grid operation regulation under the new electricity market, have become cyberphysical systems (CPSs). The essence of the CPS is to construct a closed-loop feedback system based on automatic data flow between the cyber space and the physical space [20]. Considering that the traditional model-driven control strategies may not be suitable for the cyber-physical load aggregation system, this paper presents a datadriven demand dispatch strategy consisting of state awareness, real-time analysis, scientific decision-making and precise execution between the cyber space and the physical space.

The main contributions in this paper are twofold. First, a closed-loop control architecture based on automatic data flow between the cyber space and the physical space is built to support demand dispatch in the CPS of aggregated EWHs, including state awareness, real-time analysis, scientific decision-making and precise execution. Second, based on the closed-loop control architecture, the state awareness logic, real-time analysis logic, scientific decision-making logic and precise execution logic are designed to implement demand dispatch.

The rest of this paper is organized as follows. Section 2 introduces the cyber-physical load aggregation system. Section 3 describes the related models and thermostat setpoint control rule. The data-driven demand dispatch strategy is presented in Sect. 4. Case studies are given in Sect. 5. Finally, the paper is concluded in Sect. 6.

\section{Cyber-physical load aggregation system}

Smart residential EWH appliances are cyber-physical units, which integrates the functions of perception, calculation, control and communication. The smart EWH appliances and the cloud-based platform of the load aggregator constitute a cyber-physical load aggregation system, as shown in Fig. 1. In the cyber-physical load aggregation system, the cloud-based platform of the load aggregator receives the demand dispatch event from service providers in the electricity market and perceives realtime state parameters of every EWH. Then the cloud-based platform executes the demand dispatch strategy based on the perceptual state parameters and the residential configuration information for each EWH.

Figure 2 shows the residential configuration information for each EWH by user's APP of the cloud-based platform, which includes the thermostat setpoint and the preferences for demand dispatch. The user's preferences include whether it is acceptable to participate in demand dispatch, the satisfactory setpoint range and whether it is acceptable if beyond range.

Figure 3 shows the implementation architecture of demand dispatch strategy for the cloud-based platform in the cyber-physical load aggregation system. This strategy is implemented by state awareness, real-time analysis, scientific decision-making and precise execution. It realizes the mapping of physical quantities of EWH appliances to cyber signals. The state awareness logic, real-time analysis

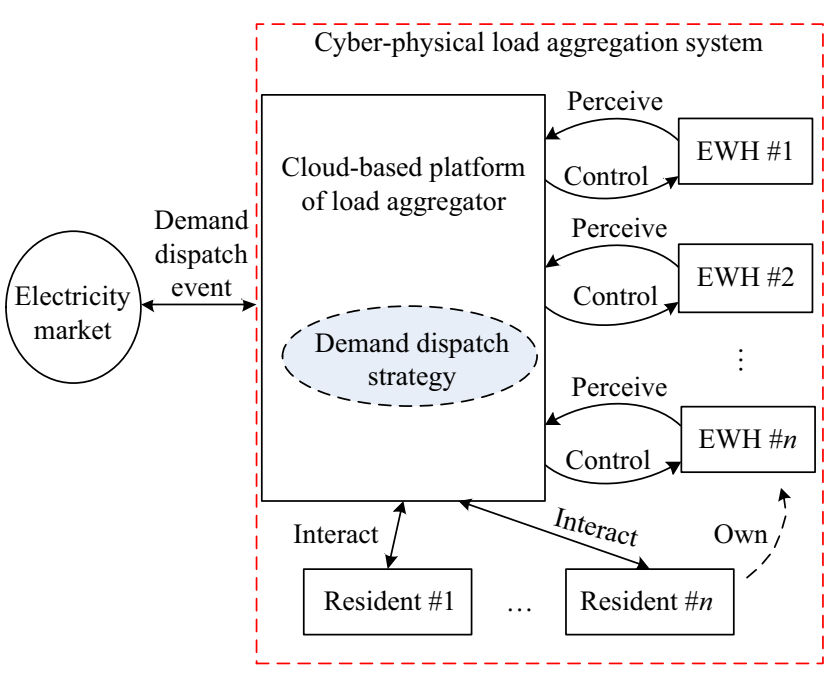

Fig. 1 Demand dispatch architecture for smart residential EWH loads 
logic, scientific decision-making logic and precise execution logic are designed in Sect. 4.

Before introducing these logics, the related models and thermostat setpoint control rule are introduced.

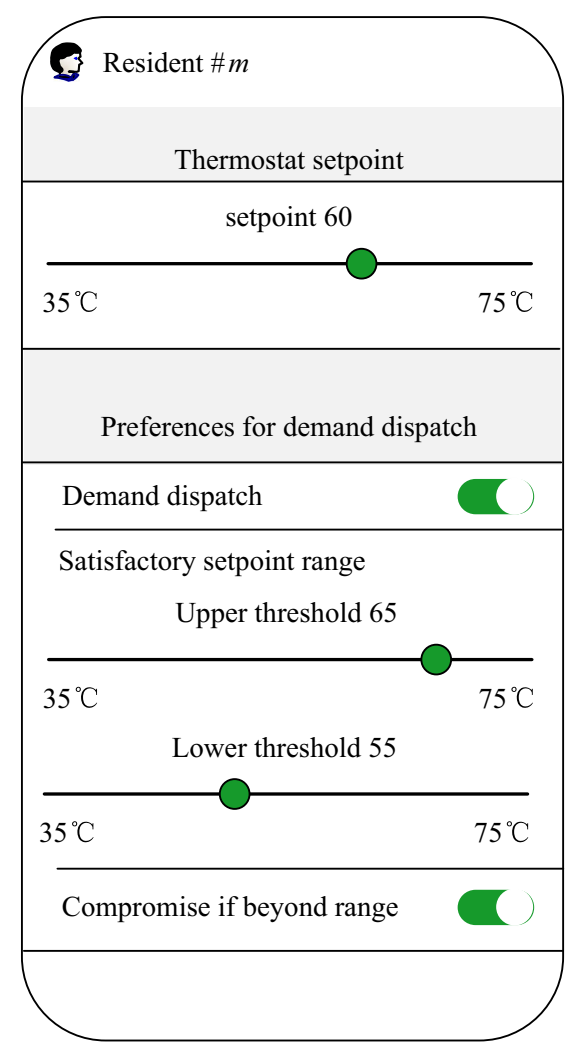

Fig. 2 Configuration information for an EWH by user's APP of cloud-based platform

\section{Related models and thermostat setpoint control rule}

In this paper, the time horizon is subdivided into equallength operational time slots. The length of the time slot is $\Delta t$.

\subsection{Multilevel incentive model}

The multilevel incentive model proposed by [14] is shown in (1), which acts as an incentive mechanism of the load aggregator to reward residents for participating in demand dispatch.

$$
R_{M k}(i)= \begin{cases}R_{1} & T_{\text {set }, L}(k) \leq T_{\text {set }}^{k}(i) \leq T_{\text {set }, U}(k) \\ R_{2} & T_{\text {set }}^{k}(i)<T_{\text {set }, L}(k), C_{\text {om }}(k)=1 \\ & \text { or } T_{\text {set }}^{k}(i)>T_{\text {set }, U}(k), C_{o m}(k)=1 \\ R_{3} \quad & T_{\text {set }}^{k}(i)<T_{\text {set }, L}(k), C_{o m}(k)=0 \\ & \text { or } T_{\text {set }}^{k}(i)>T_{\text {set }, U}(k), C_{o m}(k)=0\end{cases}
$$

where $R_{M k}(i)$ is the incentive rate for the resident \#k in time slot $i ; R_{1}$ is the level 1 incentive rate; $R_{2}$ is the level 2 incentive rate; $R_{3}$ is the level 3 incentive rate; $T_{\text {set }, L}(k)$ is the lower threshold of the satisfactory setpoint range for the resident $\# k ; T_{\text {set }, U}(k)$ is the upper threshold of the satisfactory setpoint range for the resident $\# k ; T_{\text {set }}^{k}(i)$ is the thermostat setpoint of the EWH \#k at time slot $i$; $C_{o m}(k)$ indicates whether the resident $\# k$ compromises if beyond range, 1 represents yes, 0 represents no.

For convenience, $R_{M k}(i)$ is represented as follow:

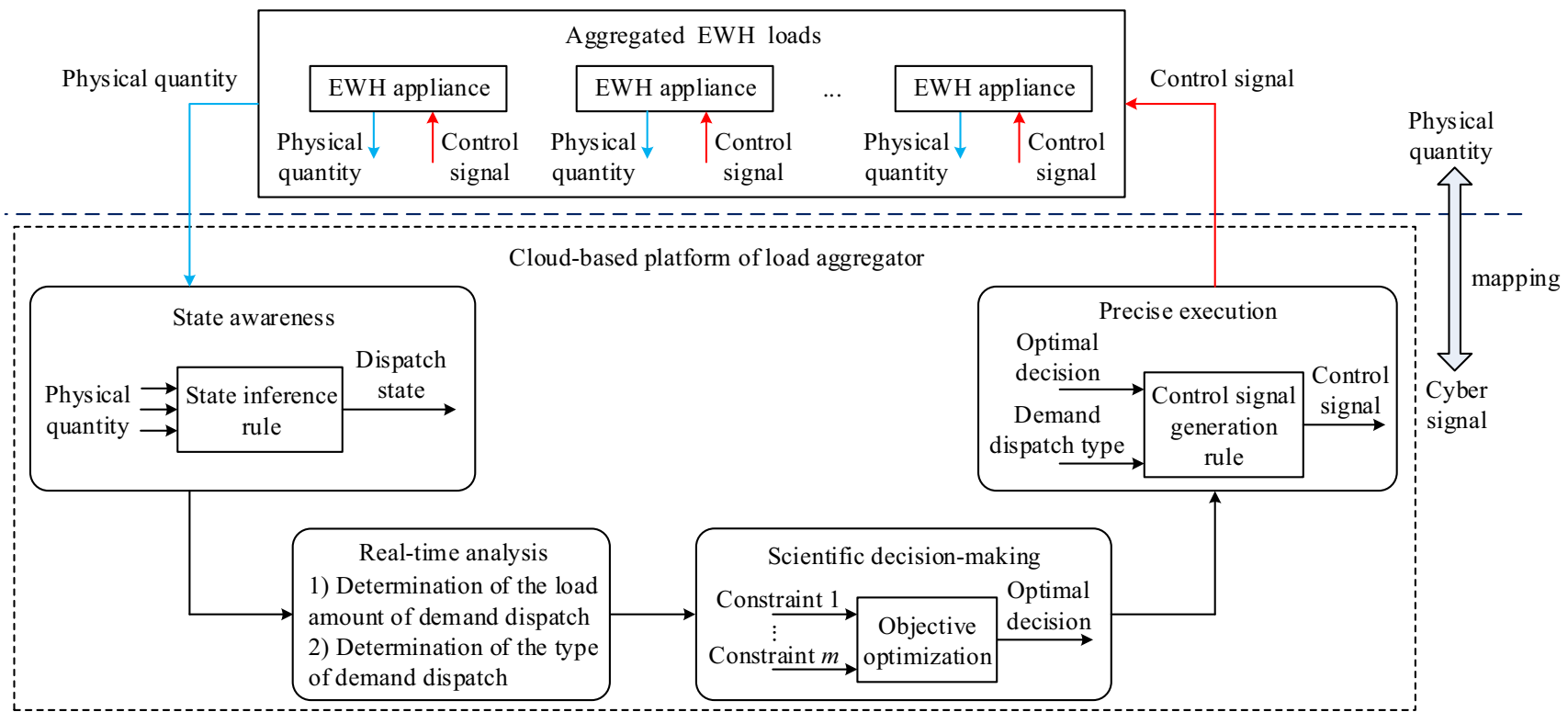

Fig. 3 Implementation architecture of demand dispatch strategy for cloud-based platform in cyber-physical load aggregation system 
$R_{M k}(i)=f_{1}\left(T_{s e t}^{k}(i), T_{s e t, L}(k), T_{s e t, U}(k), C_{o m}(k)\right)$

Figure 4 shows the flow chart of determining the incentive rate in time slot $i$ for the resident $\# k$ under the multilevel incentive model [14]. From Fig. 4, it can be observed that the more serious the degree of violating user's preference is, the higher the reward level is, namely the more the reward payment for load aggregator is.

\subsection{EWH appliance model}

The EWH appliance model proposed by [15] is shown in (3) and (5), which serves as a mapping model of the real EWH appliance in the cyber space. It includes the temperature model and internal cyber-physical control model. The temperature model describes the change rule of water temperature in the tank. The internal cyber-physical control model describes the transition rule of the heating element status. The temperature model is described as follows:

$$
\begin{aligned}
T(i+1)= & \frac{T(i)\left(V_{\text {tank }}-f_{r}(i) \Delta t\right)}{V_{\text {tank }}}+\frac{T_{\text {inlet }} f_{r}(i) \Delta t}{V_{\text {tank }}} \\
& +\left[P_{W H} \eta_{W H} S_{W H}(i) A_{1}-\frac{A_{\text {tank }}\left(T(i)-T_{a}(i)\right)}{R_{\text {tank }}}\right] \\
& \cdot \frac{\Delta t}{A_{2}} \frac{1}{V_{\text {tank }}} A_{3}
\end{aligned}
$$

where $T(i+1)$ and $T(i)$ are the water temperatures in the tank at the start of time slot $i+1$ and time slot $i$, respectively; $T_{\text {inlet }}$ is the temperature of inlet water; $f_{r}(i)$ is the hot water flow rate in time slot $i$; $V_{\text {tank }}$ is the volume of the tank; $\Delta t$ is the length of time slot; $P_{W H}$ is the rated power of the EWH; $\eta_{W H}$ is the efficiency factor; $S_{W H}(i)$ is the status of the heating element in time slot $i, 0$ represents off, 1

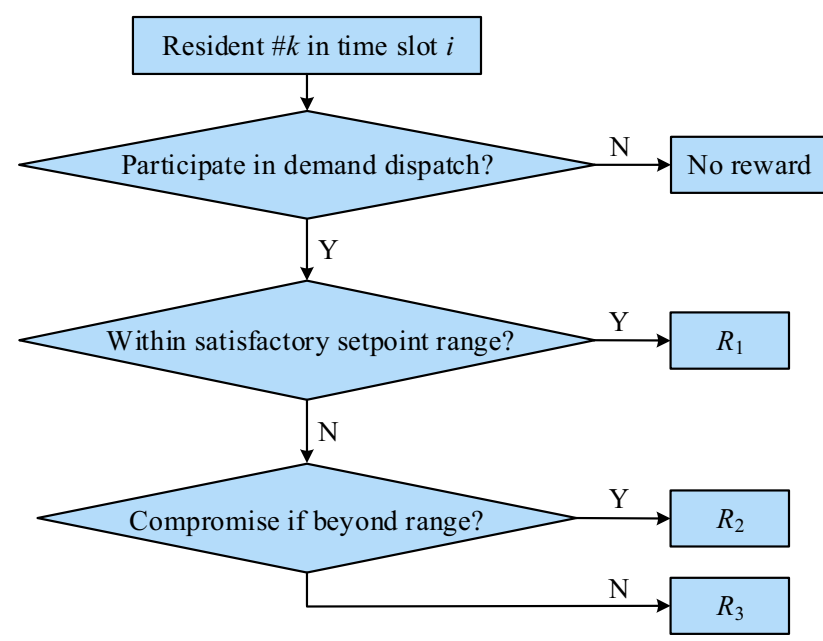

Fig. 4 Flow chart of determining incentive rate in time slot $i$ for resident \#k under the multilevel incentive model represents on; $A_{\text {tank }}$ is the surface area of the tank; $T_{a}(i)$ is the room temperature in time slot $i ; R_{\text {tank }}$ is the heat resistance of the tank; the value of $A_{1}$ is $3412 \mathrm{Btu} / \mathrm{kWh}$; the value of $A_{2}$ is $60 \mathrm{~min} / \mathrm{h}$; the value of $A_{3}$ is $1 / 8.34 \mathrm{gal} / \mathrm{lb}$.

For convenience, $T(i+1)$ is represented as follows:

$T(i+1)=f_{2}\left(T(i), S_{W H}(i), f_{r}(i), T_{a}(i)\right)$

The internal cyber-physical control model is described as follows:

$$
S_{W H}(i)= \begin{cases}0 & T(i)>T_{\text {set }}(i) \\ 1 & T(i)<T_{\text {set }}(i)-D_{s} \\ S_{W H}(i-1) & T_{\text {set }}(i)-D_{s} \leq T(i) \leq T_{\text {set }}(i)\end{cases}
$$

where $T_{\text {set }}(i)$ is the thermostat setpoint at the start of time slot $i ; D_{s}$ is the thermal insulation temperature deadband.

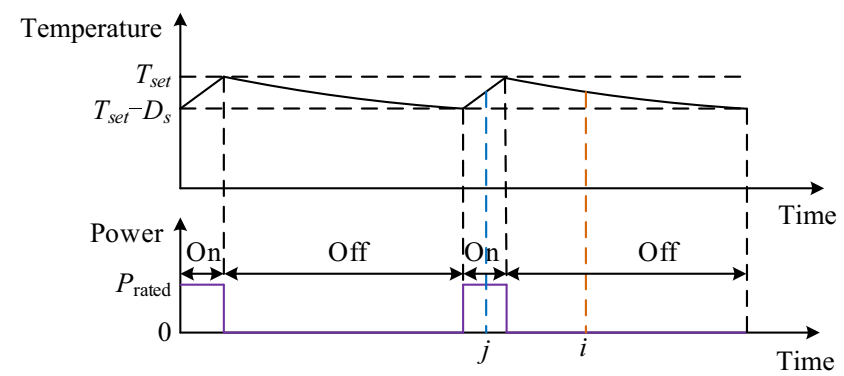

(a) Operation principle of an EWH

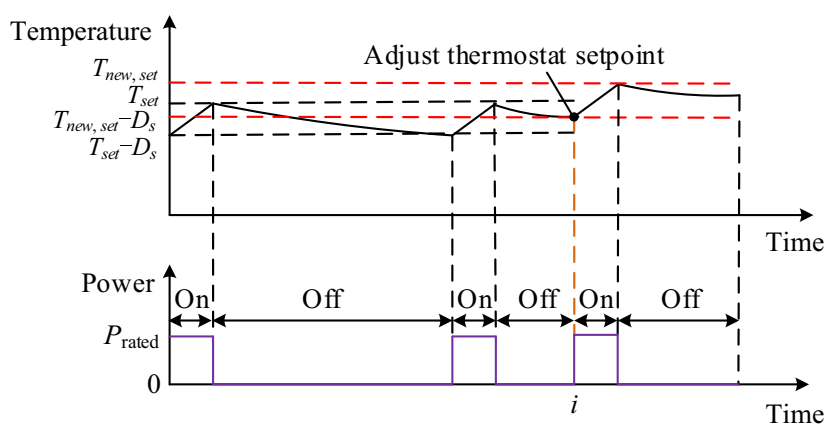

(b) Thermostat setpoint control principle from off to on

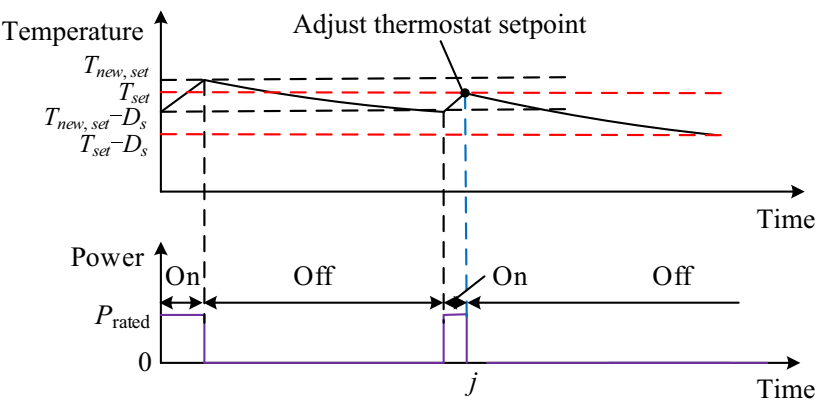

(c) Thermostat setpoint control principle from on to off

Fig. 5 Schematic diagram of operation principle of an EWH and thermostat setpoint control principle 


\subsection{Thermostat setpoint control rule}

Figure 5 shows the principle of thermostat setpoint control for an EWH. It describes the relationship between the change in thermostat setpoint and the on/off state transition for an EWH. The operation principle of an EWH is shown in Fig. 5(a), which can be verified by (5). When the hot water temperature rises to the thermostat setpoint $T_{\text {set }}$, the EWH will stop heating automatically (from on to off). When the hot water temperature drops to the temperature $T_{s e t}-D_{s}$, the EWH will start heating automatically (from off to on).

Figure 5(b) shows the thermostat setpoint control principle from off to on. According to the above operation principle of an EWH, if an EWH is changed from off state to on state at time slot $i$, the new thermostat setpoint should be set to at least $T(i)+D_{s}$. Similarly, as shown in Fig. 5(c), if an EWH is changed from on state to off state at time slot $j$, the new thermostat setpoint should be set to at most $T(j)$.

However, in the actual adjustment operation, the thermostat setpoint must be an integer. Therefore in this paper, the thermostat setpoint control rule that triggers a change in the operation state of an EWH is determined as follows:

$T_{\text {new set }}(i)= \begin{cases}\lceil T(i)\rceil+D_{s} & f_{\text {action }}(i)=1 \\ \lfloor T(i)\rfloor & f_{\text {action }}(i)=-1\end{cases}$

where $T_{\text {new,set }}(i)$ is the new thermostat setpoint at time slot $i ;\lceil X\rceil$ is the function to round the number $X$ to the upper whole number; $\lfloor X\rfloor$ is the function to round the number $X$ to the lower whole number; $f_{\text {action }}(i)$ is a function that represents action type at time slot $i$, the value of 1 means turning the unit on, the value of -1 means shutting off the unit.

\section{Demand dispatch strategy}

As shown in Fig. 3, this section presents the state awareness logic, real-time analysis logic, scientific decision-making logic and precise execution logic, which constitute the implementation logic of the data-driven demand dispatch strategy.

\subsection{State awareness logic}

State awareness logic is used to obtain the scheduling state of each EWH in time slot $i$, by the acquired data from each EWH at the start of time slot $i$. The implementation process is shown in Fig. 6. It includes the load grouping process and the scheduling state generation process.

In the load grouping process, an EWH load may be inferred to belong to one of the following three groups:

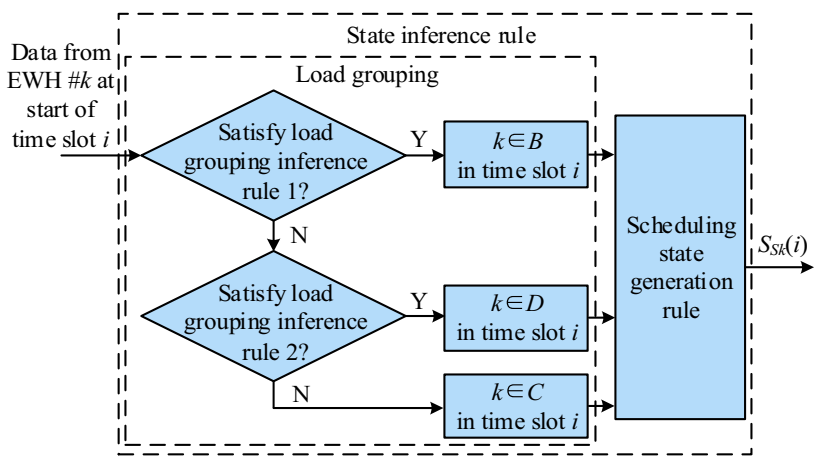

Fig. 6 Implementation process of state awareness logic

group $B$, group $C$ and group $D$. The EWH loads in the group $B$ can respond to the load increase task. The EWH loads in the group $D$ can respond to the load curtailment task. The EWH loads in the group $C$ can't respond to any task.

If an EWH load belongs to group $B$ in time slot $i$, the EWH status must be off in time slot $i$ and the new thermostat setpoint shall not exceed the allowed maximum thermostat setpoint at the start of time slot $i$. So the load grouping inference rule 1 is designed as follows: if $f_{2}\left(T_{k}(i), 0, f_{r}(i), T_{a}(i)\right) \geq T_{\text {set }}^{k}(i)-D_{s}, \quad P_{\text {art }}(k)=1$, $s_{k}(i)=0,\left\lceil T_{k}(i)\right\rceil+D_{s} \leq T_{\max }$, then $k \in B . P_{\text {art }}(k)$ indicates whether the resident \#k agrees to participate in demand dispatch, 1 represents yes, 0 represents no; $s_{k}(i)$ is the state of the heating element for the EWH \#k at the start of time slot $i, 1$ represents on, 0 represents off; $T_{k}(i)$ is the water temperature of the EWH \#k at the start of time slot $i$; $T_{\max }$ is the maximum thermostat setpoint specified by the manufacturer.

If an EWH load belongs to group $D$ in time slot $i$, the EWH status must be on in time slot $i$ and the new thermostat setpoint shall not be lower than the allowed minimum thermostat setpoint at the start of time slot $i$. So the load grouping inference rule 2 is designed as follows: if $f_{2}\left(T_{k}(i), 1, f_{r}(i), T_{a}(i)\right) \leq T_{\text {set }}^{k}(i), \quad P_{\text {art }}(k)=1, \quad s_{k}(i)=1$, $\left\lfloor T_{k}(i)\right\rfloor \geq T_{\text {min }}$, then $k \in D . T_{\text {min }}$ is the minimum thermostat setpoint specified by the manufacturer.

In the scheduling state generation process, the scheduling state of the EWH $\# k$ in time slot $i$ is generated according to the result of load grouping. The scheduling state generation rule is described as follows:

$S_{S k}(i)= \begin{cases}1 & k \in B \\ 0 & k \in C \\ -1 & k \in D\end{cases}$

where $S_{S k}(i)$ is the scheduling state of the EWH \#k in time slot $i$.

If $S_{S k}(i)=1$, it shows that the EWH \#k can respond to the load increase task in time slot $i$. If $S_{S k}(i)=0$, it shows 
that the EWH \#k can't respond to any task in time slot $i$. If $S_{S k}(i)=-1$, it shows that the EWH \#k can respond to the load curtailment task in time slot $i$.

\subsection{Real-time analysis logic}

Real-time analysis logic determines the actual load amount that is needed to be scheduled and the executed task type for the aggregated EWHs in time slot $i$. The load amount is calculated by:

$P_{S}(i)=P_{\text {event }}(i)-\left(\sum_{k=1}^{N} P_{W H}(k) s_{k}(i)-P_{\text {baseline }}(i)\right)$

where $P_{S}(i)$ is the actual load amount that is needed to be scheduled in time slot $i ; P_{\text {event }}(i)$ is the load amount specified in the demand dispatch event in time slot $i ; N$ is the total number of EWHs; $P_{W H}(k)$ is the rated power of the EWH \#k; $P_{\text {baseline }}(i)$ is the baseline load amount of the aggregated EWHs in time slot $i$, which is considered to be the load amount of the aggregated EWHs with on control in [21].

The executed task type in time slot $i$ is determined by:

$f_{T, \text { task }}(i)=\left\{\begin{array}{cc}1 & P_{S}(i)>0 \\ -1 & P_{S}(i)<0\end{array}\right.$

where $f_{T, t a s k}(i)$ is a function that represents the executed task type in time slot $i$ for the aggregated EWHs, the value of 1 means performing the load increase task, the value of -1 means performing the load curtailment task.

\subsection{Scientific decision-making logic}

Scientific decision-making logic determines the optimal combination of EWHs which are chosen to perform the task of demand dispatch during the time slot $i$, according to the results from state awareness logic and real-time analysis logic. In this paper, minimizing the total reward

Table 1 User settings for EWHs

\begin{tabular}{llllll}
\hline ID & $T_{\text {set }}\left({ }^{\circ} \mathrm{C}\right)$ & $P_{\text {art }}$ & $T_{\text {set }, L}\left({ }^{\circ} \mathrm{C}\right)$ & $T_{\text {set }, U}\left({ }^{\circ} \mathrm{C}\right)$ & $C_{\text {om }}$ \\
\hline$\# 1$ & 60 & 1 & 55 & 70 & 1 \\
$\# 2$ & 55 & 1 & 50 & 62 & 0 \\
$\# 3$ & 70 & 1 & 60 & 70 & 1 \\
$\# 4$ & 65 & 1 & 60 & 70 & 1 \\
$\# 5$ & 56 & 1 & 50 & 60 & 0 \\
$\# 6$ & 58 & 1 & 55 & 70 & 1 \\
$\# 7$ & 62 & 1 & 55 & 70 & 0 \\
$\# 8$ & 60 & 1 & 58 & 64 & 1 \\
$\# 9$ & 58 & 1 & 55 & 60 & 0 \\
\hline
\end{tabular}

payment to the residents is considered as a decision objective. So the optimization objective function during the time slot $i$ is formed as follows:

$\min \left(\sum_{k \in O_{i}} R_{M k}(i) P_{W H}(k) \theta_{k}(i)\right)$

where $O_{i}$ indicates a set of the EWH units that can participate in demand dispatch in time slot $i ; \theta_{k}(i)$ is the binary decision variable, the value of which is 0 or 1 . The value of 1 means choosing the EWH \#k to perform the task of demand dispatch during the time slot $i$. The value of 0 means not choosing the EWH \#k to perform the task of demand dispatch during the time slot $i$.

The optimization problem is subject to the constraints in (11) and (12):

$$
\begin{aligned}
& O_{i}=\left\{k \mid S_{S k}(i)=f_{T, t a s k}(i), k=1,2, \ldots, N\right\} \\
& \sum_{k=1}^{N} P_{W H}(k) \theta_{k}(i) \geq\left|P_{S}(i)\right|
\end{aligned}
$$

The above optimization problem is a $0-1$ integer programming problem, which can be solved by the optimization software tools. The optimal solution constitutes a set of scheduled EWH loads during the time slot $i$, which is denoted by $S_{i}$ :

$S_{i}=\left\{k \mid \theta_{k}(i)=1, k \in O_{i}\right\}$

\subsection{Precise execution logic}

Precise execution logic generates the control signal, which includes the new thermostat setpoints at time slot $i$ for the scheduled EWH loads. According to the thermostat setpoint control rule specified in (6), the control signal generation rule is described as follows:

$T_{\text {new }, \text { set }}^{k}(i)= \begin{cases}\left\lceil T_{k}(i)\right\rceil+D_{s} & k \in S_{i}, f_{T, \text { task }}(i)=1 \\ \left\lfloor T_{k}(i)\right\rfloor & k \in S_{i}, f_{T, \text { task }}(i)=-1\end{cases}$

where $T_{\text {new,set }}^{k}(i)$ is the new thermostat setpoint of the EWH $\# k$ at time slot $i$.

\subsection{Description of demand dispatch strategy}

The data-driven demand dispatch strategy for state awareness, real-time analysis, scientific decision-making and precise execution at the start of a time slot $i$ is summarized as follow:

1) State awareness: for each EWH \#k, obtain the scheduling state $S_{S k}(i)$.

2) Real-time analysis: determine the load amount $P_{S}(i)$ and the executed task type $f_{T, \text { task }}(i)$.

3) Scientific decision-making: solve $\theta_{k}(i)$ according to (10)-(12) and form the scheduled EWH load set $S_{i}$. 
Table 2 Physical attributes and initial status for nine EWHs

\begin{tabular}{llllllc}
\hline ID & $T_{\text {ini }}\left({ }^{\circ} \mathrm{C}\right)$ & $s_{\text {ini }}$ & $P_{\mathrm{WH}}(\mathrm{kW})$ & $V_{\text {tank }}(\mathrm{gallon})$ & $R_{\text {tank }}\left({ }^{\circ} \mathrm{F} \cdot \mathrm{ft}^{2} \cdot \mathrm{h} / \mathrm{Btu}\right)$ \\
\hline$\# 1$ & 58.5 & Off & 2 & 60 & 20 & 3 \\
$\# 2$ & 53.2 & On & 3 & 60 & 18 & 3 \\
$\# 3$ & 69.0 & Off & 2 & 60 & 3 \\
$\# 4$ & 62.8 & Off & 3 & 90 & 18 & 3 \\
$\# 5$ & 53.6 & On & 2 & 60 & 20 & 3 \\
$\# 6$ & 56.2 & On & 3 & 90 & 18 & 3 \\
$\# 7$ & 60.8 & Off & 3 & 60 & 18 & 3 \\
$\# 8$ & 58.4 & On & 2 & 60 & 20 \\
\hline
\end{tabular}

4) Precise execution: for each EWH $k \in S_{i}$, generate the new thermostat setpoint $T_{n e w, s e t}^{k}(i)$ and send the control signal to the EWH \#k.

\section{Case studies}

The proposed demand dispatch strategy is performed on both a nine-resident system and some much larger systems, and several case studies are designed.

The first case study is carried out in a nine-resident system, which is designed to illustrate the effectiveness and advantages of the proposed demand dispatch strategy, since more detailed information can be demonstrated in a smallscale case study. The second case study is carried out in the 800 -resident system. The results show that the proposed demand dispatch strategy is not only applicable to the small residential system but also for the large residential system. The third case study is used to show changes in total reward costs with the number of residents, the length of event time and the load-scheduling amount. These three case studies illustrate the effectiveness and the advantages of the proposed strategy as well as excellent scalability. In addition, note that in this paper, a resident has only an EWH and the length of event time is subdivided into short equal-length operational time slots. The length of the short time slot is $1 \mathrm{~min}$.

\subsection{First case study}

The test system has nine residents. In this system, every resident has different personal preferences for their own EWHs, as shown in Table 1. The physical attributes and the initial status for the nine EWHs are given in Table 2. $T_{\text {ini }}$ stands for the water temperature in the tank at initial time, $s_{\text {ini }}$ stands for the state of heating element at initial time. And suppose that the residents won't use hot water during this period. The room temperature is $18^{\circ} \mathrm{C}$. The reward rates $R_{1}, R_{2}$, and $R_{3}$ are set to 4,8 , and 12 cents/ (kW·min), respectively [14].

In the nine-resident case study, the cloud-based platform of the load aggregator received the demand dispatch event from a service provider at initial time, which asked the load aggregator to increase $4 \mathrm{~kW}$ for 5 min among these nine EWHs. The power and temperature change of each EWH are analyzed under no-control and the proposed strategy. Also, the total reward costs are compared under different control schemes.

\section{1) Results under no-control}

Figure 7 shows the power change versus time and the temperature change versus time under no-control during the period of the event. From Fig. 7(a), it can be observed that at the start of time slot 1 , the EWHs \#1, \#3, \#4, \#7, and \#9 could participate in demand dispatch. From Fig. 7(b), it can be observed that the temperature change of the EWHs conforms to the operating rule of an EWH appliance described in Sect. 3.3, which illustrates that the simulation is reliable.

2) Results under the proposed strategy

Figure 8 shows the power change versus time and the temperature change versus time under the proposed strategy during the period of the event. Table 3 gives the control scheme under the proposed control. From Table 3, it can be showed that during the period of the event, the EWHs \#1 and \#7 are controlled to respond to demand dispatch at the start of time slot 1 , which can be demonstrated by comparing Figs. 7 and 8 .

Figure 9 compares the power change of the test system versus time under no control and the proposed strategy. The power of the load aggregation system can be calculated by summing up the power of all the EWHs in the test system. Figure 9 shows that compared with the power under no control, the power of the load aggregation system 

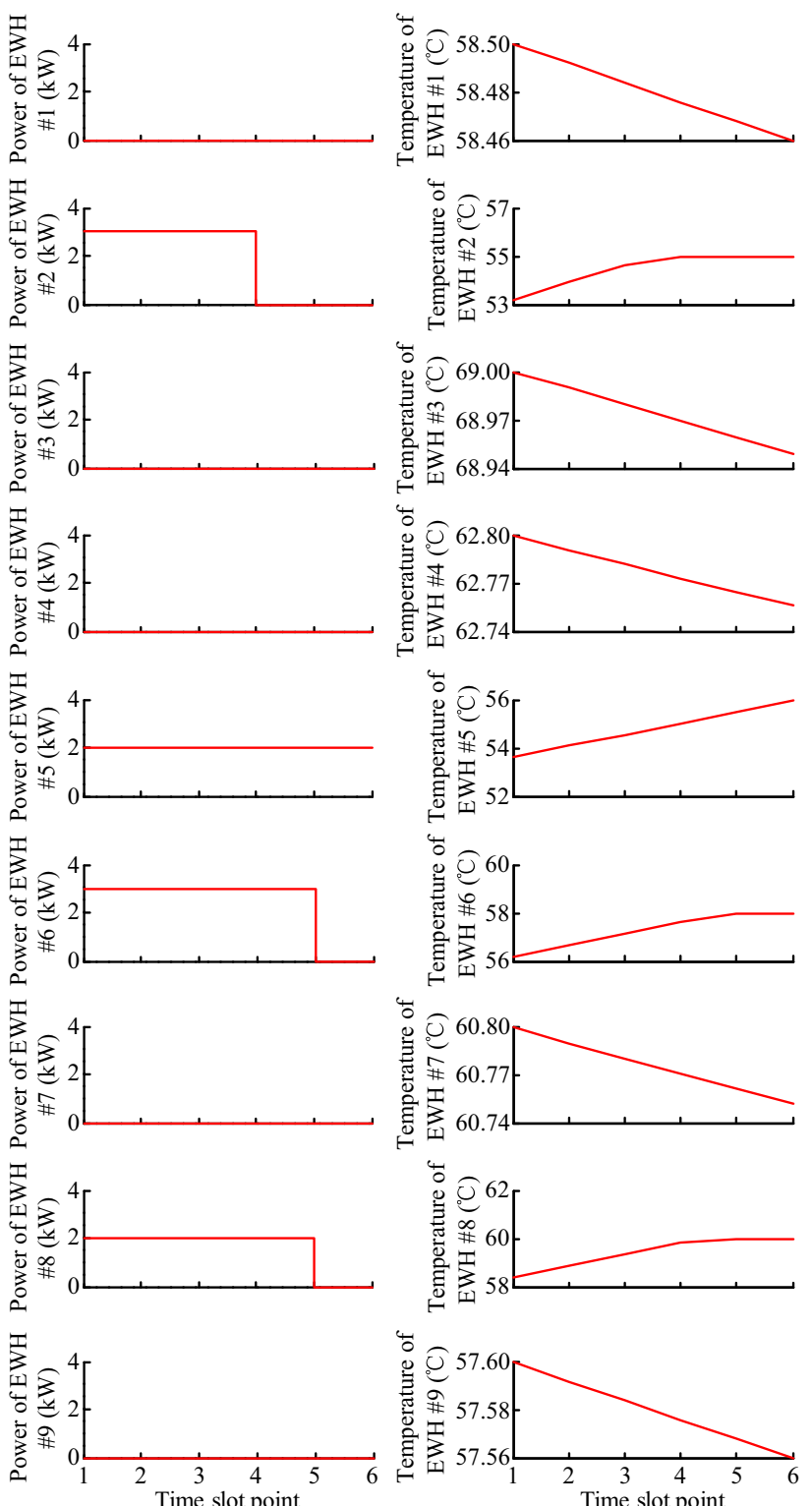

(a) Power change versus time
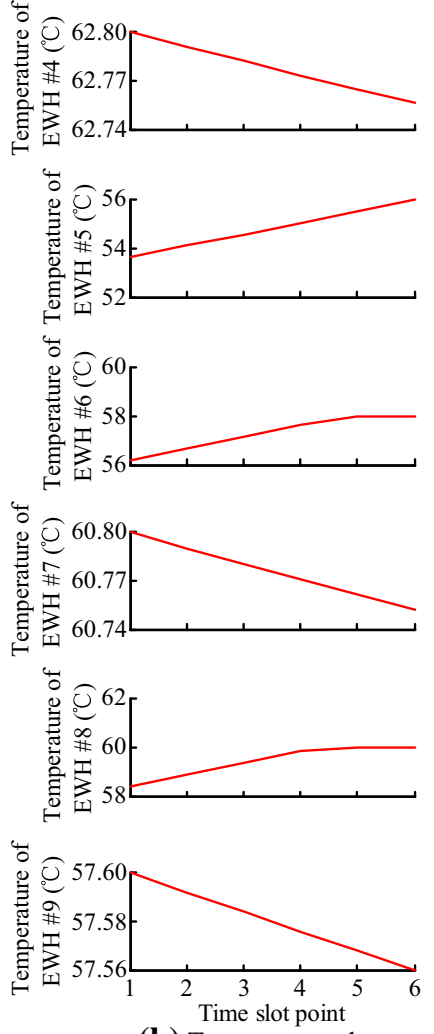

(b) Temperature change versus time

Fig. 7 Simulation under no-control during period of event

is increased by $5 \mathrm{~kW}$ during the period of the event. It shows that the load aggregator completes the task specified in the demand dispatch event, which proves that the proposed strategy is effective.

\section{3) Comparison of reward costs}

Table 4 gives the list of control schemes meeting the requirement for time slot points $1-5$. Figure 10 compares the reward payments under different control schemes. From Fig. 10, it can be observed that the reward payment under the control scheme No. 3 is minimal. The control scheme No. 3 is generated under the proposed strategy, which shows that the proposed strategy can minimize the

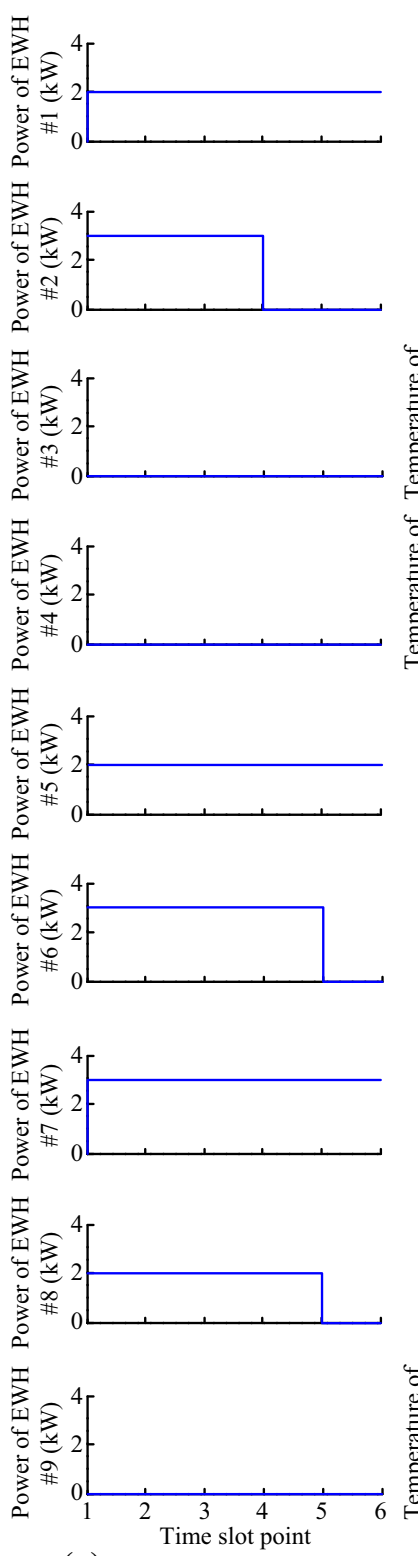

(a) Power change versus time
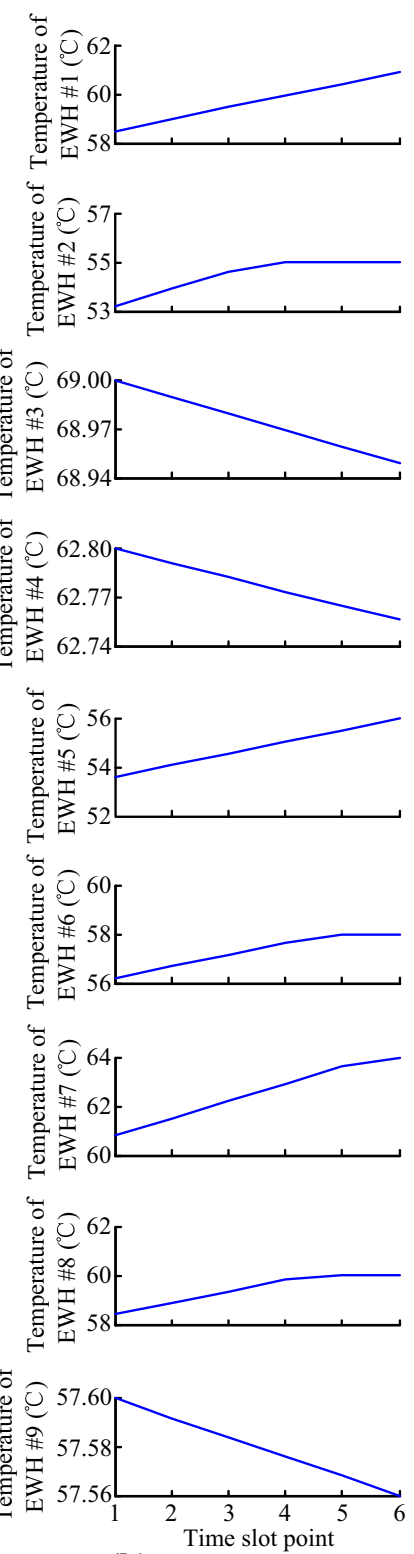

(b) Temperature change

versus time
Fig. 8 Simulation under the proposed strategy during period of event

Table 3 Control scheme under the proposed strategy

\begin{tabular}{ll}
\hline Time slot point & Control scheme \\
\hline 1 & $(\# 1, \# 7)$ \\
2 & No control \\
3 & No control \\
4 & No control \\
5 & No control \\
\hline
\end{tabular}

reward payment for the load aggregator. The reason is that the new thermostat setpoints of the EWHs \#1 and \#7 after 


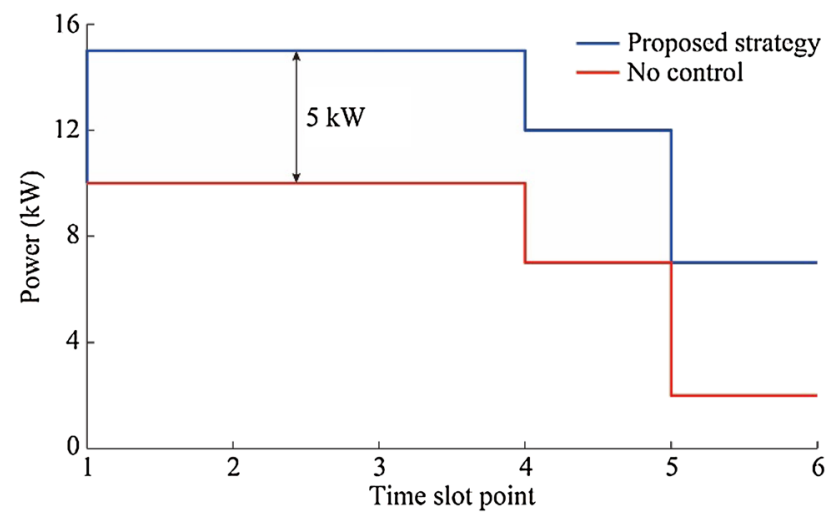

Fig. 9 Power change of load aggregation system versus time in first case study

Table 4 List of control schemes meeting the requirement

\begin{tabular}{|c|c|c|c|c|c|}
\hline \multirow[t]{2}{*}{ No. } & \multicolumn{5}{|c|}{ Control scheme } \\
\hline & 1 & 2 & 3 & 4 & 5 \\
\hline 1 & $(\# 1, \# 3)$ & No control & No control & No control & No control \\
\hline 2 & $(\# 1, \# 4)$ & No control & No control & No control & No control \\
\hline 3 & $(\# 1, \# 7)$ & No control & No control & No control & No control \\
\hline 4 & $(\# 1, \# 9)$ & No control & No control & No control & No control \\
\hline 5 & $(\# 3, \# 4)$ & No control & No control & No control & No control \\
\hline 6 & $(\# 3, \# 7)$ & No control & No control & No control & No control \\
\hline 7 & $(\# 3, \# 9)$ & No control & No control & No control & No control \\
\hline 8 & $(\# 4, \# 7)$ & No control & No control & No control & No control \\
\hline 9 & $(\# 4, \# 9)$ & No control & No control & No control & No control \\
\hline 10 & $(\# 7, \# 9)$ & No control & No control & No control & No control \\
\hline
\end{tabular}

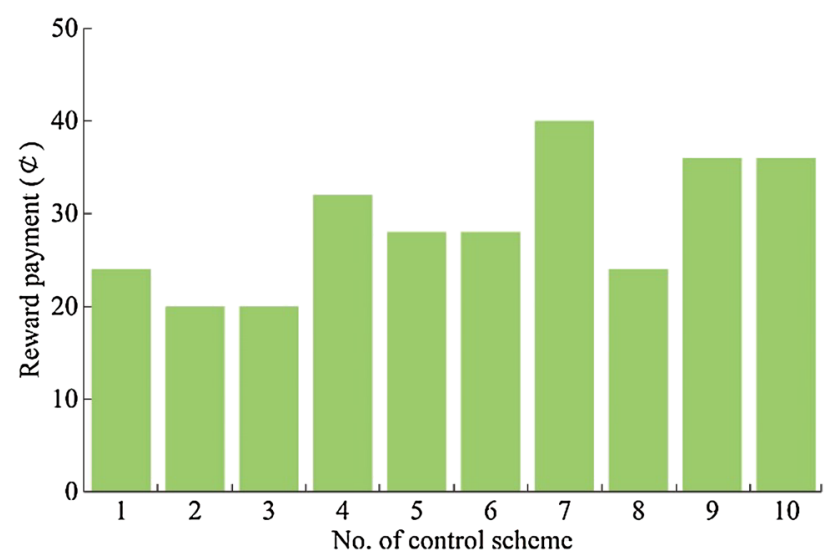

Fig. 10 Reward payments under different control schemes

being adjusted are still within satisfactory setpoint range, so the reward rate for residents \#1 and \#7 is $R_{1}$.

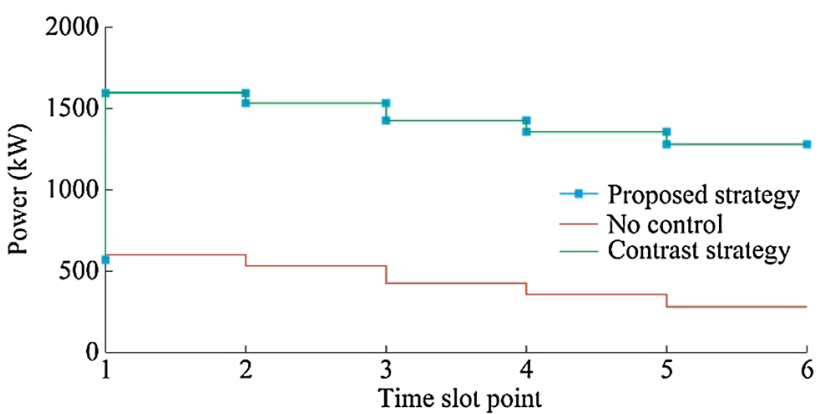

(a)

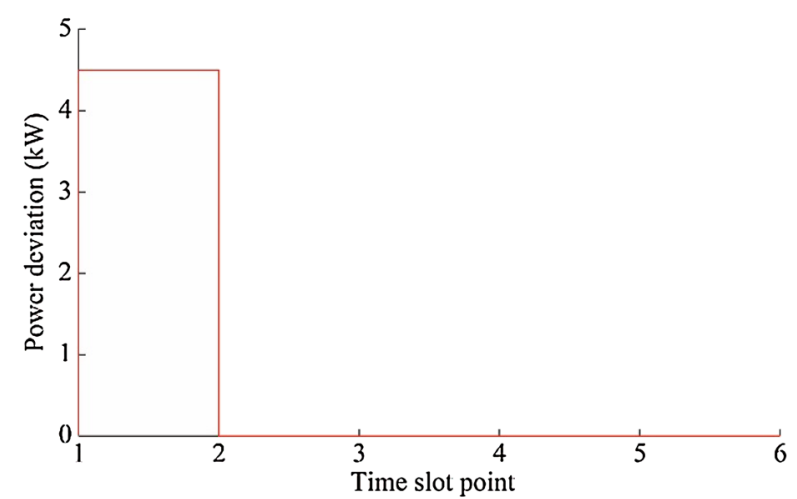

(b)

Fig. 11 Power change of load aggregation system versus time in second case study

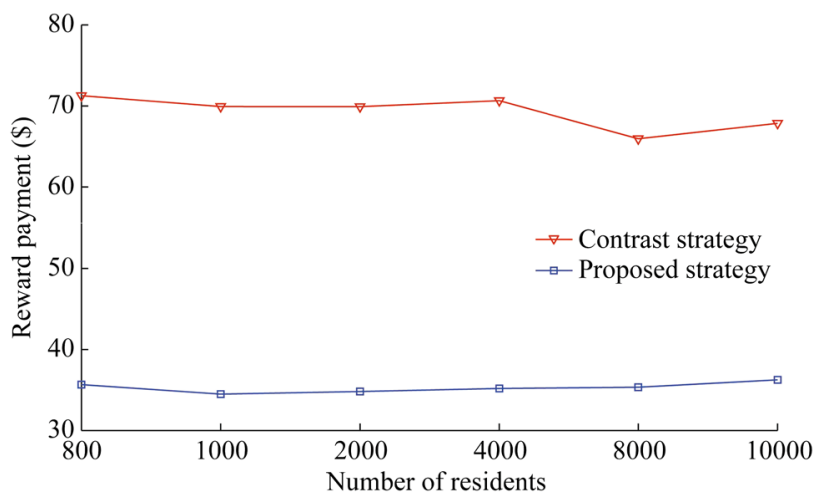

Fig. 12 Changes in reward payment versus different numbers of residents

\subsection{Second case study}

The second case is studied in a large system which has 800 residents, because the proposed strategy is expected to solve practical problems within this scale. In the largescale system study, the load aggregator is asked to increase $1 \mathrm{MW}$ for 5 min among these EWHs.

A strategy which is to minimize the difference between the actual dispatched load amount and the load amount specified in the demand dispatch event [22], is used as a contrast strategy. And we compare the proposed strategy 


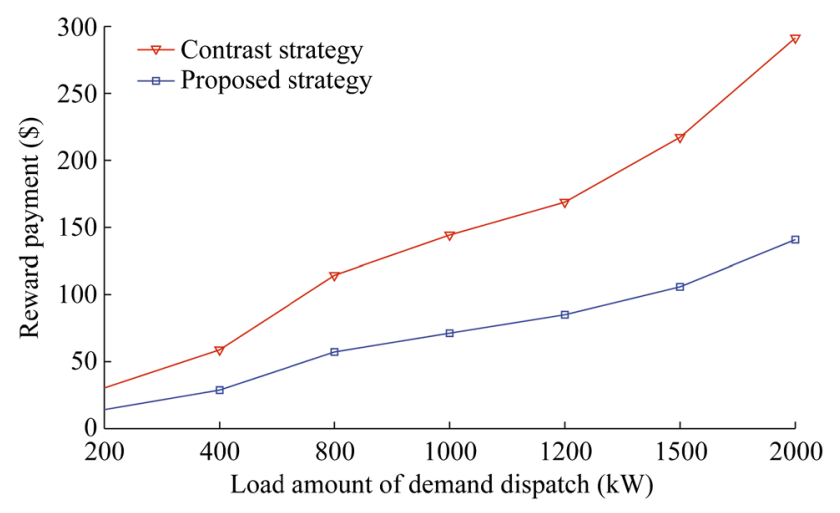

Fig. 13 Changes in reward payment versus different dispatched load amount

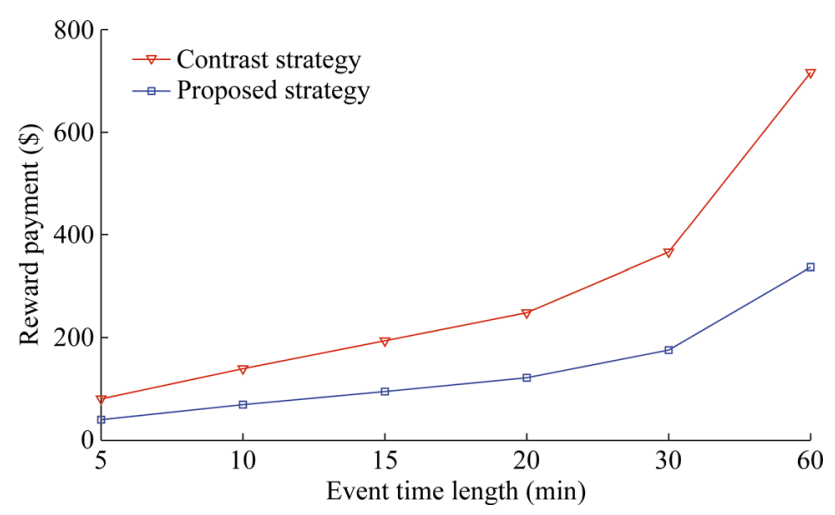

Fig. 14 Changes in reward payment versus different event time lengths

with the contrast strategy from the total reward payment and the power change of the test system.

The reward payments under the proposed strategy and the contrast strategy in the second case study are 48.9 dollars and 83.3 dollars, respectively. Figure 11(a) shows the power change of the load aggregation system versus time in the second case study. Figure 11(b) shows the power deviation change versus time between the power under the proposed strategy and the power under the contrast strategy. From Fig. 11, it can be concluded that compared with the contrast strategy, the proposed strategy can realize the minimum reward payment of the load aggregator, and completes the requirement from the service provider. It also shows that the proposed strategy can solve practical problems within 800 residents.

\subsection{Third case study}

In order to further illustrate the advantages and the good scalability of the proposed strategy, the third case is studied with different numbers of residents, different dispatched load amount and different event time length, respectively.
We compare the results under the proposed strategy and the contrast strategy.

Figure 12 gives the changes in reward payment versus different numbers of residents, when the load aggregator is asked to increase $500 \mathrm{~kW}$ for $10 \mathrm{~min}$. From Fig. 12, it can be observed that the reward payment under the proposed strategy is always less than the one under the contrast strategy. It shows that the proposed strategy can realize the minimum reward payment of the load aggregator with different numbers of residents.

Figure 13 gives the changes in reward payment versus different dispatched load amount in a 4000-resident system for $10 \mathrm{~min}$. From Fig. 13, it can be observed that the reward payment under the proposed strategy is less and the difference between the reward payment under the proposed strategy and under the contrast strategy increases with the increase of the dispatched load amount. It shows that the proposed strategy can realize the minimum reward payment of the load aggregator with different dispatched load amount.

Figure 14 gives the changes in reward payment versus different event time length in a 4000-resident system for increasing $1 \mathrm{MW}$. From Fig. 14, it can be observed that the reward payment under the proposed strategy is less, and the difference between the reward payment under the proposed strategy and under the contrast strategy increases with the increase of the event time length. It shows that the proposed strategy can realize the minimum reward payment of the load aggregator with different event time length.

\section{Conclusion}

In this paper, the demand dispatch in cyber-physical load aggregation system is studied. Based on the theory of CPS, a control strategy consisting of state awareness, realtime analysis, scientific decision-making and precise execution between the cyber space and the physical space is proposed to automatically respond to the supply-demand regulation of power grid for the load aggregator. The results of the three different study cases show the effectiveness, advantage, and excellent scalability of the proposed strategy. The proposed strategy can be helpful for load aggregator, to achieve the optimal economic control of residents' EWHs for responding to supply-demand regulation of power grid.

Acknowledgements This work was supported by National Natural Science Foundation of China (No. 51777068) and Fundamental Research Funds for the Central Universities (No. 2016XS15).

Open Access This article is distributed under the terms of the Creative Commons Attribution 4.0 International License (http:// creativecommons.org/licenses/by/4.0/), which permits unrestricted 
use, distribution, and reproduction in any medium, provided you give appropriate credit to the original author(s) and the source, provide a link to the Creative Commons license, and indicate if changes were made.

\section{References}

[1] Zhang J, Sun W, Wang T (2015) Studies on requirements and architecture for automated demand response system. Proc CSEE 35(16):4070-4076

[2] Zeng L, Sun Y, Ye Q et al (2017) A centralized demand response control strategy for domestic electric water heater group based on appliance cloud platform. IEEJ Trans Electr Electron Eng 12(16):16-22

[3] Gao C, Liang T, Li H et al (2013) Development and application of open automated demand response. Power Syst Technol 37(3):692-698

[4] Kris K, Daan S, Muhajir T et al (2013) Feasibility of employing domestic active demand for balancing wind power generation. In: Proceedings of the 10th international conference on European energy market, Stockholm, Sweden, 27-31 May 2013

[5] Johanna LM, Maryam K, John L et al (2015) Arbitraging intraday wholesale energy market prices with aggregations of thermostatic loads. IEEE Trans Power Syst 30(2):763-772

[6] Brooks A, Lu E, Dan R et al (2010) Demand dispatch. IEEE Power Energy Mag 8(3):20-29

[7] Elghitani F, Zhuang W (2017) Aggregating a large number of residential appliances for demand response applications. IEEE Trans Smart Grid. https://doi.org/10.1109/TSG.2017.2679702

[8] Booysen MJ, Engelbrecht JAA, Molinaro A (2013) Proof of concept: large-scale monitor and control of household water heating in near real-time. In: Proceedings of the international conference on applied energy, Pretoria, South Africa, 1-4 July 2013, 8 pp

[9] Pourmousavi SA, Patrick SN, Nehrir MH (2014) Real-time demand response through aggregate electric water heaters for load shifting and balancing wind generation. IEEE Trans Smart Grid 5(2):769-778

[10] Nazir MS, Galiana FD, Prieur A et al (2016) Unit commitment incorporating histogram control of electric loads with energy storage. IEEE Trans Power Syst 31(4):2857-2866

[11] Ernest Orlando Lawrence Berkeley National Laboratory (2009) Open automated demand response communications specification (version 1.0). Report Number: CEC-500-2009-063/LBNL$1779 \mathrm{E}$

[12] OpenADR Alliance (2011) OpenADR 2.0 profile specification A profile. http://www.openadr.org. Accessed 10 October 2012

[13] OpenADR Alliance (2012) OpenADR 2.0 profile specification B profile. http://www.openadr.org. Accessed 10 July 2013

[14] Hu Q, Li F, Fang X et al (2017) A framework of residential demand aggregation with financial incentives. IEEE Trans Smart Grid 9(1):497-505

[15] Shao S, Pipattanasomporn M, Rahman S (2013) Development of physical-based demand response-enabled residential load models. IEEE Trans Power Syst 28(2):607-614

[16] Shaad M, Moneni A, Diduch CP et al (2012) Parameter identification of thermal models for domestic electric water heaters in a direct load control program. In: Proceedings of the IEEE conference on electrical and computer engineering, Montreal, Canada, 29 April-2 May 2012, 5 pp

[17] Lu N, Chassin DP (2004) A state-queueing model of thermostatically controlled appliances. IEEE Trans Power Syst 19(3):1666-1673

[18] Lu N, Zhang Y (2013) Design considerations of a centralized load controller using thermostatically controlled appliances for continuous regulation reserves. IEEE Trans Smart Grid 4(2):914-921

[19] Wang D, Fan M, Jia H (2014) User comfort constraint demand response for residential thermostatically-controlled loads and efficient power plant modeling. Proc CSEE 34(13):2071-2077

[20] National Standardization Management Committee (2017) White paper on cyber-physical system. China

[21] Heffner GC, Goldman CA, Moezzi MM (2006) Innovative approaches to verifying demand response of water heater load control. IEEE Trans Power Deliv 21(1):388-397

[22] Qi YB, Wang D, Jia HJ et al (2015) Research on demand response for thermostatically controlled appliances based on normalized temperature extension margin control strategy. Proc CSEE 35(21):5455-5464

Lukun ZENG received the B.S. degree in communication engineering from North China Electric Power University, Beijing, China, in 2009, where he is currently working toward the Ph.D. degree. His research interests include demand side management and cyberphysical system in smart grid.

Yi SUN received the M.S. degree in communication and information system and Ph.D. degree in electric information technology from North China Electric Power University, Beijing, China, in 2009 and 2014, respectively. He is currently a professor with information and communication engineering in North China Electric Power University. His mainly research interests include smart power consumption, demand response, and power system communication technology.

Xingjie ZHOU received her B.S. degree in electrical engineering from Changzhou University, Changzhou, China. Now She is working towards the M.S. degree in electronic and information engineering in North China Electric Power University. Her research interests include demand response in cyber-physical load aggregation system.

Bin LI received the Ph.D. degree from Beijing University of Posts and Telecommunications, Beijing, China. Currently he is a professor in the Institute of Electrical Engineering in North China Electric Power University. His research interests include power system automation and information technology, demand side management, cyber-physical system in smart grid.

Bing QI is a professor in the Institute of Electric and Electronic Engineering and also director of Communication Laboratory in North China Electric Power University. $\mathrm{He}$ is also an expert in the Committee of IEC PC118 Smart Grid User Interface Project and a committee member of SAC/TC549 National Smart Grid User Interface Standardization Technology Committee. His current research interests include the communication technology in electric power system, the analysis and progressing of electrical information, demand response. 